\title{
Magnetic Domain Imaging of Ni-Mn-Ga Heusler Alloys Using Lorentz TEM
}

\author{
Isha Kashyap ${ }^{1}$, Marc De Graef ${ }^{1}$ \\ 1. Dept. of Materials Science and Engineering, Carnegie Mellon University, Pittsburgh PA, USA
}

Ni-Mn-Ga alloys are Ferromagnetic Shape Memory Alloys (FSMAs) in which a high Magnetic Field Induced Strain (MFIS) can be obtained by inducing the rearrangement of martensite twin variants under the influence of an external magnetic field. A very high recoverable strain of $9.5 \%$ has been observed in $\mathrm{Ni}-\mathrm{Mn}-\mathrm{Ga}$ alloys [1], and because of this property, they find applications in actuators and sensors. NiMn-Ga alloys are multi-ferroic materials exhibiting more than one ferroic property, in this case, ferromagnetism and ferroelasticity. Moreover, the alloy also goes through an atomic ordering at a temperature below $800^{\circ} \mathrm{C}$. All these properties give rise to interesting features in this alloy, namely twin variants and twin boundaries; anti-phase boundaries (APBs); magnetic domains and domain walls. However, there is a lack of magnetic microstructure studies of this alloy at a nanometer scale. In addition, the interaction between various microstructural defects and magnetic domains in this alloy is poorly understood. In this study, we utilize the Lorentz Transmission Electron Microscopy (LTEM) technique to investigate the magnetic domain microstructure and interaction between different ferroic domains in Ni-Mn-Ga alloys. The Fresnel or out-of-focus Lorentz mode is a simple way of imaging magnetic domain walls. When an electron passes through a region of magnetic material with a magnetic field, it experiences a Lorentz force and as a result gets deflected in a specific direction. Only the inplane magnetic induction will contribute to the deflection. This deflection gives rise to a region of deficiency of electrons or a region of excess of electrons at the locations of magnetic domain walls which in turn appears as a dark feature or a bright feature in the resulting image. Three different images are taken in the Fresnel mode namely: in-focus, under-focus and over-focus image. When the lens goes from under-focus to over-focus mode, the contrast of walls is reversed. The Fresnel mode LTEM only provides qualitative information about the domain walls. However, quantitative information about the magnetic induction can be extracted from the Fresnel series of images by doing phase reconstruction based on transport-of-intensity equation. The phase reconstructed color map gives the direction of integrated magnetic induction inside the sample. A detailed description of Lorentz technique and phase reconstruction can be found in [2].

In this study, the magnetic domain structure of a $\mathrm{Ni}_{50} \mathrm{Mn}_{29} \mathrm{Ga}_{21}$ single crystal in its demagnetized state was investigated in the Lorentz mode TEM with a FEI Tecnai F20 microscope with a dedicated Lorentz pole piece. The sample was in the ordered austenitic state at room temperature. Figures 1(a)-(c) show a Fresnel through-focus series of images. The magnetic structure contains $180^{\circ}$ domain walls as shown by white arrows in Fig. 1(b). The curved dark features in the images are bend-contours arising from diffraction effects. No magnetic contrast was observed in the in-focus image. Also note the reversal of contrast of domain walls in over-focused and under-focused images. The type of magnetic domain rearrangement is dependent on the relative orientation of the easy axis of magnetization with respect to the thin foil normal. Straight domain walls are indicative of the in-plane magnetization direction. The magnetic induction orientation in a particular region of the thin foil was obtained using a phase reconstruction method. Fig. 1(d) shows the phase reconstructed color map. The different colors on the color map represent domains with different magnetic induction direction. For example, the blue color on the color map represent domains whose magnetization point towards the top and yellow color represents the domains whose magnetization point towards the bottom. Intermediate colors correspond to induction 
directions containing both horizontal and vertical components. The color map in Fig. 1(d) shows that the magnetization direction changes by $180^{\circ}$ across the domain walls. A curved feature with white and black contrast was also observed crossing a $180^{\circ}$ domain wall. This is believed to be the contrast associated with anti-phase boundaries as reported by Venkateswaran et al. [3]. It was also observed that the double fringe contrast reversed after crossing the domain wall (shown by black arrow in Fig. 1(c)). The unusual magnetic contrast is because of the antiferromagnetic nature of APBs. It can be seen from the color map (Fig. 1(d)) that the APBs have a different magnetization direction as compared to the surrounding matrix. Fig. 1(e) shows another example of interaction between APBs and $180^{\circ}$ domain walls in a martensitic $\mathrm{Ni}_{50} \mathrm{Mn}_{29} \mathrm{Ga}_{21}$ alloy. Black arrows point to various APBs while white arrows point to $180^{\circ}$ domain walls. Fig. 1(f) shows an under-focus image taken from the same alloy. The black arrows point to two types of fine twin bands. One of the fine twin bands is enclosed by a large twin variant which shows a maze-like domain structure in the image. A maze-like domain structure is indicative of an outof-plane magnetization direction. In the near future, Electron Channeling Contrast Imaging (ECCI), which is a non-destructive technique for studying near-surface defects, will be employed to study twin boundaries and other defects in bulk shape memory Heusler alloys. The results from this ECCI study will be compared to LTEM and TEM observations [4].

\section{References:}

[1] A. Sozinov et al, Applied Physics Letters 80 (2002), p.1746.

[2] M. De Graef, Introduction to conventional transmission electron microscopy (2003).

[3] S. P. Venkateswaran, N. T. Nuhfer, and M. De Graef, Acta Materialia 55 (2007), p.2621.

[4] The authors acknowledge the support by an NSF grant, DMR-1306296 and use of the Materials Characterization Facility at Carnegie Mellon University supported by grant MCF-677785.

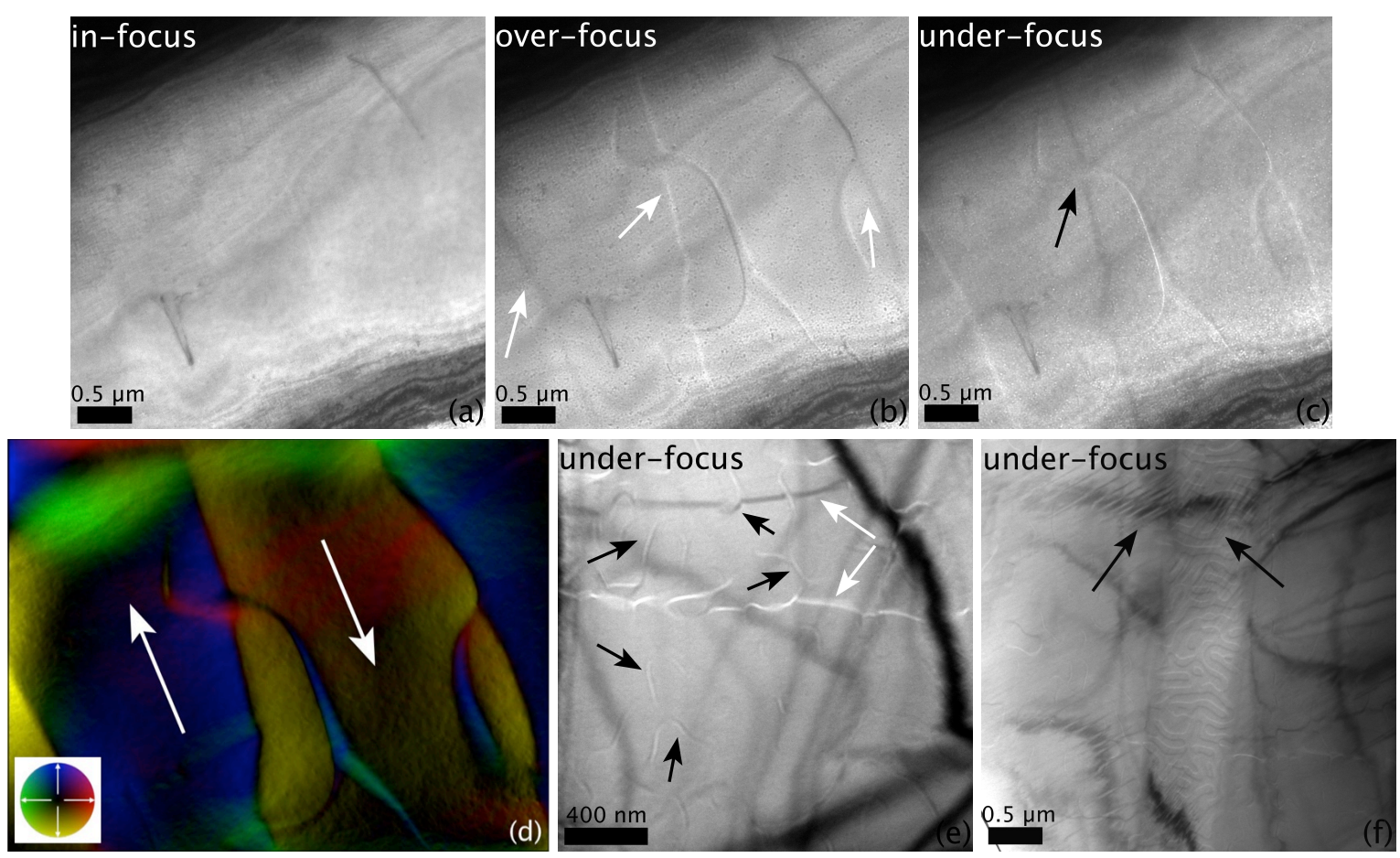

Figure 1. (a)-(c) Fresnel series of images of austenitic $\mathrm{Ni}_{50} \mathrm{Mn}_{29} \mathrm{Ga}_{21}$ alloy, (d) Phase reconstructed color map, (e) Under-focus image of a martensitic $\mathrm{Ni}_{50} \mathrm{Mn}_{29} \mathrm{Ga}_{21}$ alloy showing APBs and $180^{\circ}$ domain walls, (f) Under-focus image showing twin variants and maze-like magnetic domain structure. 